\title{
Spiral Waves in a Lattice Array of Josephson Junction Chaotic Oscillators with Flux Effects
}

\author{
Balamurali Ramakrishnan, ${ }^{1}$ Ramesh Ramamoorthy, ${ }^{1}$ Chunbiao Li, ${ }^{2,3}$ Akif Akgul, ${ }^{4}$ \\ and Karthikeyan Rajagopal $\mathbb{D}^{5}$ \\ ${ }^{1}$ Chennai Institute of Technology, Chennai, Tamilnadu, India \\ ${ }^{2}$ Jiangsu Collaborative Innovation Center of Atmospheric Environment and Equipment Technology (CICAEET), \\ Nanjing University of Information Science \& Technology, Nanjing 210044, China \\ ${ }^{3}$ Jiangsu Key Laboratory of Meteorological Observation and Information Processing, \\ Nanjing University of Information Science \& Technology, Nanjing, China \\ ${ }^{4}$ Department of Electrical and Electronics Engineering, Faculty of Technology, Sakarya University of Applied Sciences, \\ Serdivan 54050, Sakarya, Turkey \\ ${ }^{5}$ Nonlinear Systems and Applications, Faculty of Electrical and Electronics Engineering, Ton Duc Thang University, \\ Ho Chi Minh City, Vietnam
}

Correspondence should be addressed to Karthikeyan Rajagopal; karthikeyan.rajagopal@tdtu.edu.vn

Received 8 September 2020; Accepted 31 December 2020; Published 13 January 2021

Academic Editor: Adel Ouannas

Copyright ( $) 2021$ Balamurali Ramakrishnan et al. This is an open access article distributed under the Creative Commons Attribution License, which permits unrestricted use, distribution, and reproduction in any medium, provided the original work is properly cited.

Josephson junction devices play a significant role in various physical nonlinear systems because of their complex characteristics. Chaotic phenomenon in various types of Josephson junction devices has been widely reported, but many of those literature studies exempted the analysis into multistability and megastability features of the device. In this work, we investigate the network behaviour using a type of Josephson junction-memristor (JJM) device considering the feedback flux effects while modelling. We have considered both AC- and DC-type external excitation currents, and while considering the AC excitation, the system shows megastability (Ramakrishnan et al. 2020). When analysing the lattice layer network constructed with JJM excited by DC bias current, the network shows a turbulent behaviour thus forming spiral waves. This was not the case when we applied AC bias current for which the network showed a much pattern-like formation confirming localised areas of energy distribution. This energy distribution is due to the homogeneous states of the local nodes which are correlated by the respective periodicity plots. When we apply AC bias current with very low frequency, the network shows small areas of local spirals which are soon dissipated by the inhomogeneous nodes nearby. Thus, we could show that the external bias current plays an important role in the collective performance of the Josephson junction devices.

\section{Introduction}

A mathematical model of a superconducting Josephson junction finds its attraction among nonlinear dynamical studies because of its rich variety of characteristics. Mostly, the nonlinear dynamical systems, nonlinearity, and the corresponding parameter region are significant to reveal chaotic behaviour $[1,2]$. Chaotic systems are more sensitive to the initial condition for particular parameter values.
Negative resistor, nonlinear capacitor, and inductor could be used to induce nonlinearity [3]. Indeed, prologue of the memristor in the Josephson junction circuit engendered the nonlinear behaviour $[1,3]$, that is, the dependency of memductance on input current and memory effect enhance the transition between different modes of oscillations in the circuit [4]. Resetting initial values of the states may lead to different attractor profiles and results in transition from periodic to complex chaotic oscillation $[4,5]$. 
In biological systems such as neuronal activity in the neocortex $[6,7]$, cardiac tissue $[8,9]$, and pancreatic beta cells [10], reaction-diffusion systems $[11,12]$, and a network of coupled oscillators $[13,14]$, an interesting phenomenon is observed and identified as spatiotemporal patterns. Spiral waves represent remarkable spatiotemporal structures, and they appear while the spatiotemporal system is not in a thermodynamically equilibrium state. The collective behaviour and pattern formation in a spatiotemporal system caused by the network topology depend on the dynamics of a single building block in the network [15]. On the contrary, the topological defect can be identified with the movement of the spiral wave tip (singularity in the core) [16]. Coexistence of synchronization and nonsynchronization is another significant property studied in spatiotemporal systems. Spiral waves can be caused in a subexcitable medium, while the plane waves are interrupted with the electric field [15-20]. Spiral waves can be ascribed by calculating the spatial distribution [21]. Dynamics of spiral waves in an excitable medium is more related to the interaction of different boundaries [22]. In [23], the shape and the rotation frequency of spiral waves and, more importantly, the algorithm for selection with suitable excitation are discussed. The same concepts are experimentally studied in [24, 25].

Memory properties of the memristor (exponential flux) in the Josephson junction induce spiral wave formation because of the high nonlinear relation between charge and magnetic flux [15]. Already, we investigated the local dynamics of the Josephson junction-memristor (JJM) oscillator with exponential flux and reported spiking behaviour. Now, in this paper, our investigation focused on network behaviours.

The paper summarises the collective dynamics of the Josephson junction with exponential flux memristor. Firstly, we considered the JJM with static bias current and investigated the wave propagation in the network. In the next section, we considered a time-varying bias current and investigated the wave propagation in the JJM network. In every section, corresponding spatiotemporal plots are presented.

\section{Collective Dynamics of JJM}

In [26], the Josephson junction with magnetic effects is modelled using an exponential flux-controlled memristor along with a Josephson junction device [27] whose circuit model is given as in Figure 1.

Various dynamical properties are discussed in [26], where the authors showed infinite coexisting attractors when the excitation current is a periodic force. The mathematical model of the circuit in Figure 1 proposed by [26] is given by

$$
\begin{aligned}
\dot{x} & =\frac{1}{\beta_{C}}\left(I_{\text {Bias }}-G x-\sin (y)\right)-z-k_{0} x W(\phi), \\
\dot{y} & =x, \\
\dot{z} & =\frac{1}{\beta_{L}}(x-z), \\
\dot{\phi} & =k_{1} x-k_{2} \phi, \\
W(\phi) & =\alpha^{\beta \phi} k \beta \ln (\alpha),
\end{aligned}
$$

where the dimensionless state variables are $x=V / I_{J} R_{1}, y=\gamma / I_{L} R_{1}$, and $z=I_{L} / I_{j J}$. For the details about the parameters and the detailed derivation of the mathematical model, the readers are encouraged to refer [26]. As we have already investigated the local dynamics of the Josephson junction-memristor (JJM) circuit in [26], our interest is now to analyse the network performance of the JJM. Hence, we construct the 2D lattice network of the JJM whose mathematical model is defined by

$$
\begin{aligned}
\dot{x}_{i j}= & \frac{1}{\beta_{C}}\left(I_{\text {Bias }}-G x_{i j}-\sin \left(y_{i j}\right)-z-k_{0} x_{i j} W\left(\phi_{i j}\right)\right. \\
& +D\left(x_{i+1 j}+x_{i-1 j}+x_{i j+1}+x_{i j-1}-4 x_{i j}\right)+F(t) \beta_{i \delta_{1}} \beta_{j \delta_{2}}, \\
\dot{y}_{i j}= & x_{i j}, \\
\dot{z}_{i j}= & \frac{1}{\beta_{L}}\left(x_{i j}-z_{i j}\right), \\
\dot{\phi}_{i j}= & k_{1} x_{i j}-k_{2} \phi_{i j}, \\
W\left(\phi_{i j}\right)= & \alpha^{\beta \phi_{i j}} k \beta \ln (\alpha) .
\end{aligned}
$$

In model (2), we considered the $2 \mathrm{D}$ network with $i, j=1: N$, where $N=150$, and the external force (stimuli) applied to the network is considered to be a sinusoid defined as $F(t)=A \sin (\Omega t)$. The external force is applied to the centre of the network by choosing $i=\theta_{1}=75$ and $j=\theta_{2}=$ 75 for which $\beta_{i \theta_{1}}=\beta_{j \theta_{2}}=1$. We have considered no-flux boundary conditions for the analysis, and the initial conditions for the nodes in the network are [0.1, 0.1,0.1]. The parameters for the simulation are taken from [26], and the source current is the control variable for our discussion. We have considered two different cases of source current, and when $I_{\text {Bias }}=1.26$, it is considered a DC-excited JJM, and for $I_{\text {Bias }}=I \sin (\omega t)$ with $I=2.5$ and $\omega=1.2$, the model is considered an AC-excited JJM. 


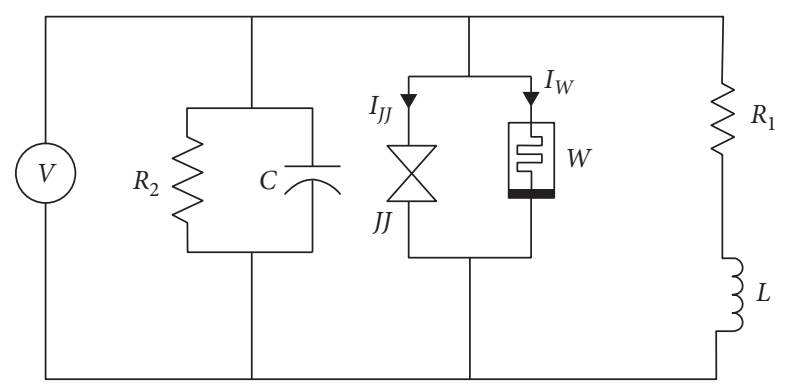

Figure 1: The Josephson junction with magnetic effects modelled using an exponential flux-controlled memristor [26].

2.1. Considering DC Bias Current. In the earlier work in [26], the JJM with DC bias current does not have complex behaviours such as multistability and coexistence, but it shows spiking behaviour for certain values of the bias current $\left(I_{\text {Bias }}\right)$, and hence, we started our discussion by studying the behaviour of JJM network (2) for various values of $I_{\text {Bias }}$. The applied external force is considered to be applied to the centre of the network, and the amplitude and frequency of the force are $A=1$ and $\Omega=0.01$. For values of $I_{\text {Bias }}<1$, the network goes unbounded, and we could not identify the wave propagation or pattern formation in the network, but for $I_{\text {Bias }}=1$, the network shows signs of spiral waves propagating to all corners of the network. This is because the periodic behaviour of the node changes drastically with the applied force which makes certain nodes to have chaotic oscillations while some to have transient-like behaviour which soon becomes periodic. To show this, we have shown the spatiotemporal dynamics of the state as well as their periods in Figure 2. The upper plots in Figure 2 show the dynamics of the network, while the lower plots show their periodic behaviours. When we increased $I_{\text {Bias }}$ to 1.2 , the spiral waves and the ring-like circular cluster formed earlier (for $I_{\text {Bias }}=1$ ) are now dissipated, and the clusters are converted into pools of incoherent nodes which are initially influenced by the external force at the entry point of the stimulus but soon the network dissipates them due to high periodicity of the nodes. This can be seen from Figure 2 for $I_{\text {Bias }}=1.2$, where the petal-like waves formed by the network show red (closer red) colour showing high period due to the high-amplitude nodes, whereas the entry being affected by the external force and amplitudes are now reduced thus showing less periods of oscillations. For $I_{\text {Bias }}=1.3$, the network is influenced fully by the external force, but the chaotic nodes are in random coherency which obstructs the wave propagation, and the network shows complete more of synchronous behaviour which can also be seen from the periodic plot ( $\max$ and min period difference reduces). The centre of the periodic plot is having high periods of oscillation confirming complex chaos, whereas the boundaries show low periods which confirms complete incoherency. Finally, for $I_{\text {Bias }}=1.4$, the network nodes are coherent, and hence, the wave propagation is not supported by the nodes as they are in complete synchronisation. This makes the external force to get dissipated in the centre of the network with only few nodes disturbed.

Now, to study the influence of the applied periodic force on the network, we consider different amplitudes of the force, while the bias current $I_{\text {Bias }}=1$, and the frequency of the periodic force is $\Omega=0.0001$. We divide the discussion into two sets, wherein in the first set, we consider higher amplitudes, and in the second set, we consider lower amplitudes. When the applied force amplitude is $A=1$, the network shows multiple small-amplitude spiral waves which are largely affected by the lower periods of oscillations in the nodes as in Figure 3. This can be seen from the periodic plot shown. When the amplitude is increased further, the low periodic oscillations of the nodes (yellow-red in the periodic plot) reduce, and for $A=7$, they completely disappear showing only complex chaotic oscillations (magenta-blue in the periodic plot). The respective spatiotemporal behaviour is now showing predominant spiral waves with much stronger amplitude features.

In the second set, lower amplitudes of periodic force are considered. For the amplitude $A=0.1$, the applied force travels through the network without much obstructions, and thus, no spiral seeds are seen, but we could see regions of cripples between $i \in(60,80)$ and $j \in(60,80)$, and these cripples are because of the low periodicity of the nodes which can be seen from the periodicity plots shown in Figure 4. When we decrease the amplitude to $A=0.01$, the propagation frequency of the target wave decreases, and we could see larger bandwidth of periods in the network. While most of the nodes remain in periods around 250 (green), the wave-captured nodes are with much higher periods around 400. This larger difference in the periods obstructs the target wave to propagate in higher velocities and thus eliminates the spiral wave transformations in the network. This can be concluded by further reducing the amplitude to $A=0.001$, where the target wave is now reduced to only two regions and the period bandwidth now increases further.

2.2. Considering AC Bias Current. After showing that the JJM network with static (DC) bias current shows spiral wave turbulence, our interest now falls on the dynamic bias current (AC). For this, we consider the bias current as a periodic sinusoid defined by $I_{\text {Bias }}=I \sin (\omega t)$. For this discussion, we consider the bias current amplitude (I) and frequency $(\omega)$ as the control parameters. Firstly, we consider two values of frequency $(\omega)$ : one as low as $\omega=0.0001$ and the other to be $\omega=1.3$. For both these cases, we vary the amplitude of bias current and study the network behaviours as shown in Figures 5 and 6. 

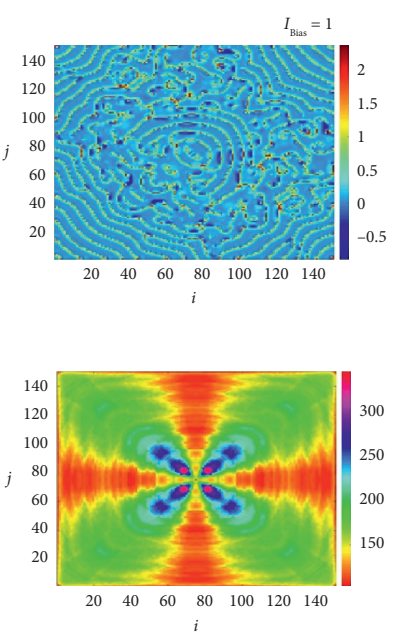
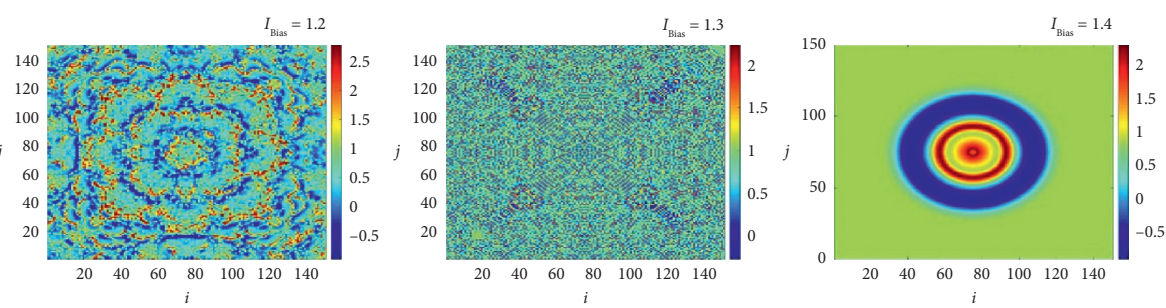

(a)

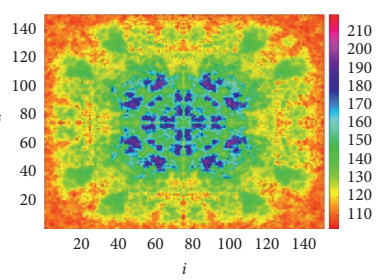

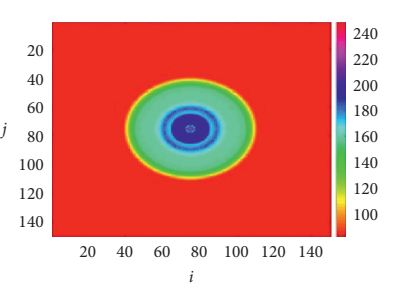

(b)

FIGURE 2: (a) The spatiotemporal plots of the nodes ( $i$ vs. $j$ ) for different values of $I_{\text {Bias }}$. (b) The periodicity of each node plotted for respective $I_{\text {Bias }}$ values.

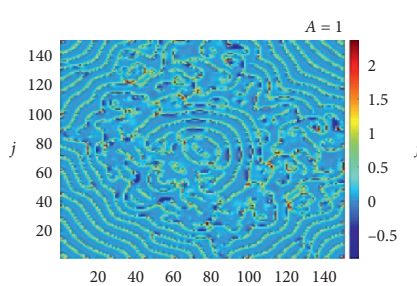

$\begin{array}{lllllll}20 & 40 & 60 & 80 & 100 & 120 & 140\end{array}$

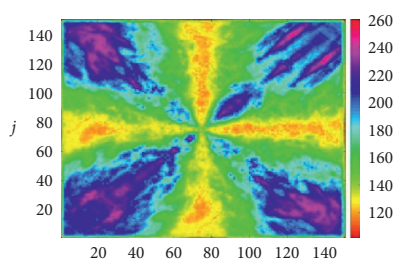

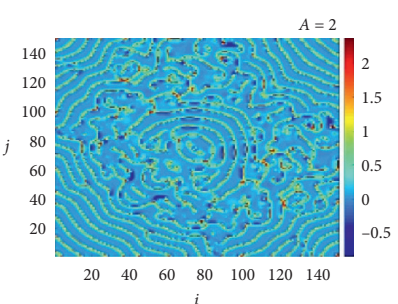

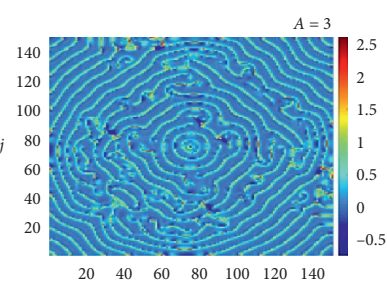

(a)

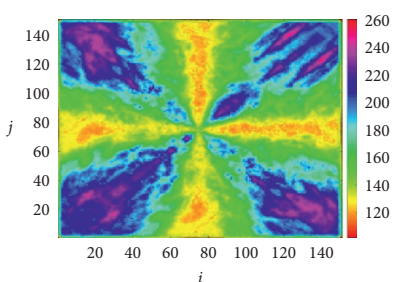

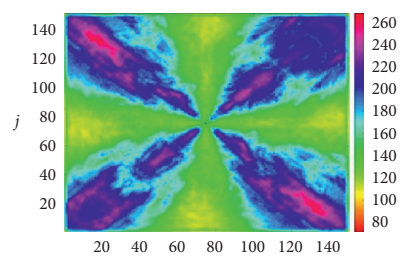

(b)
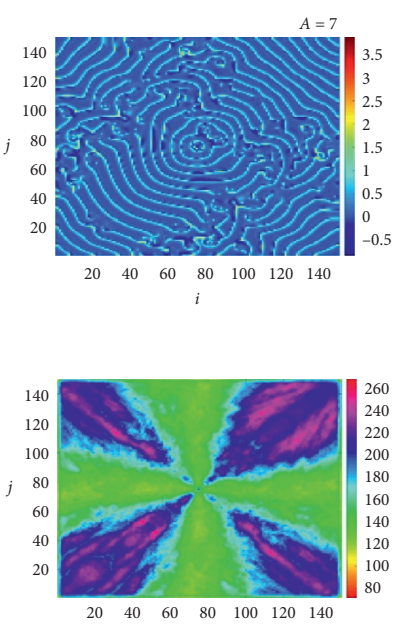

Figure 3: (a) The spatiotemporal plots of the nodes ( $i$ vs. $j$ ) for different values of periodic force amplitude $A$. (b) The periodicity of each node plotted for respective $A$ values.
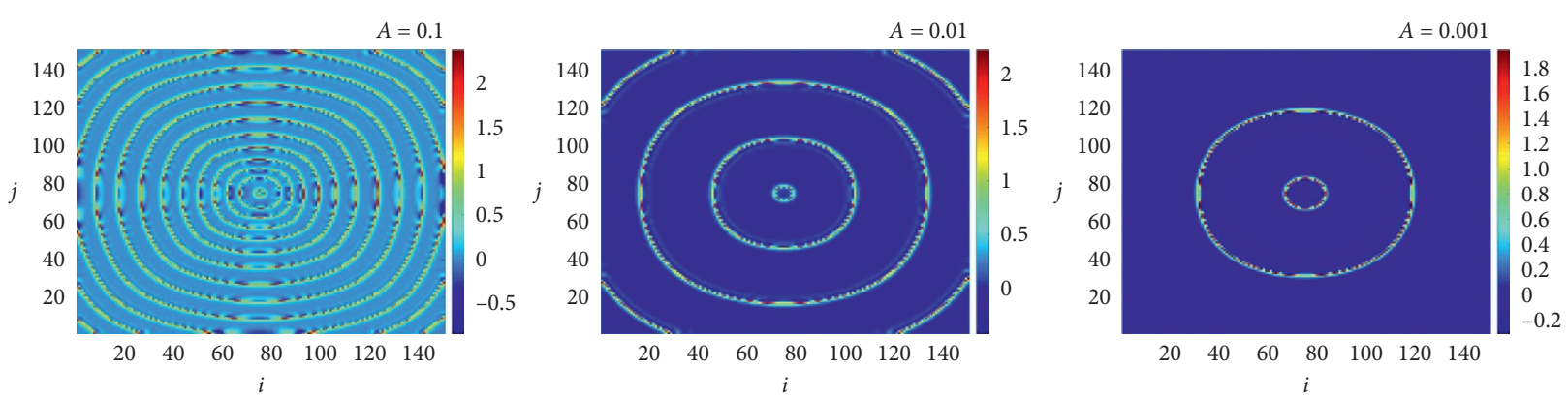

(a)

FIgURE 4: Continued. 

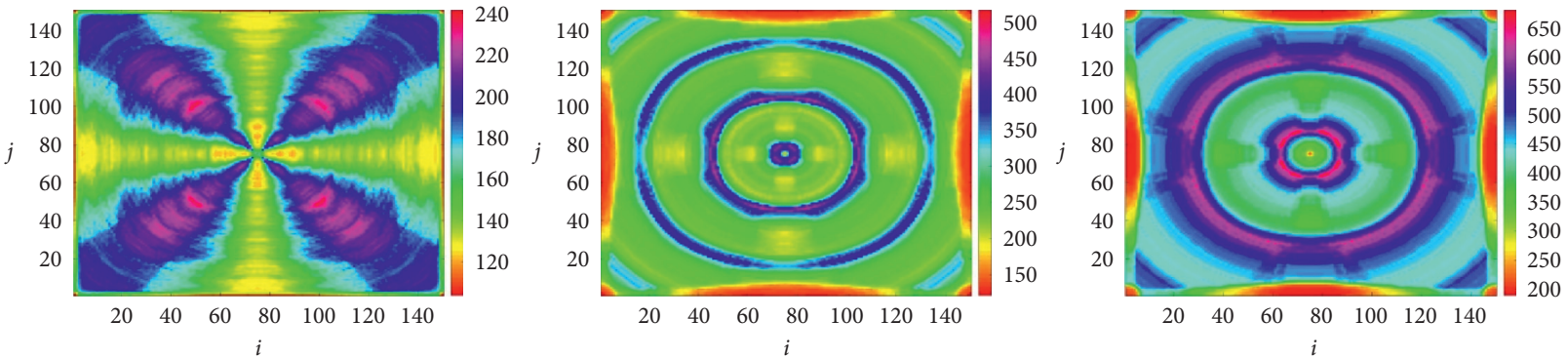

(b)

Figure 4: (a) The spatiotemporal plots of the nodes ( $i$ vs. $j$ ) for different values of periodic force amplitude $A$. (b) The periodicity of each node plotted for respective $A$ values.
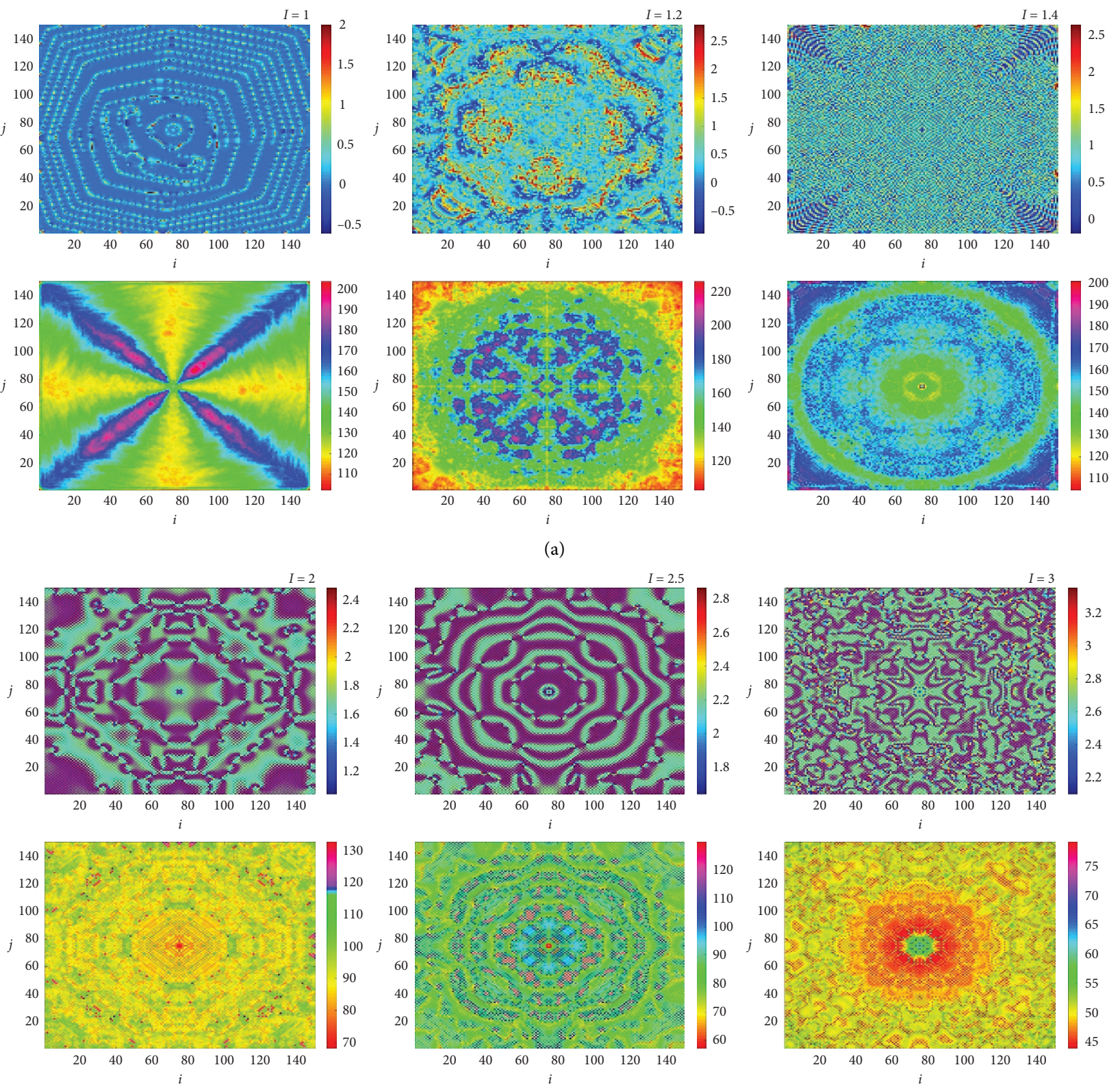

(b)

Figure 5: (a) Dynamics of the network for various values of the bias current amplitude (I). (b) The corresponding periodicity of each node for different $I$. The bias current frequency is kept as $\omega=0.0001$. 

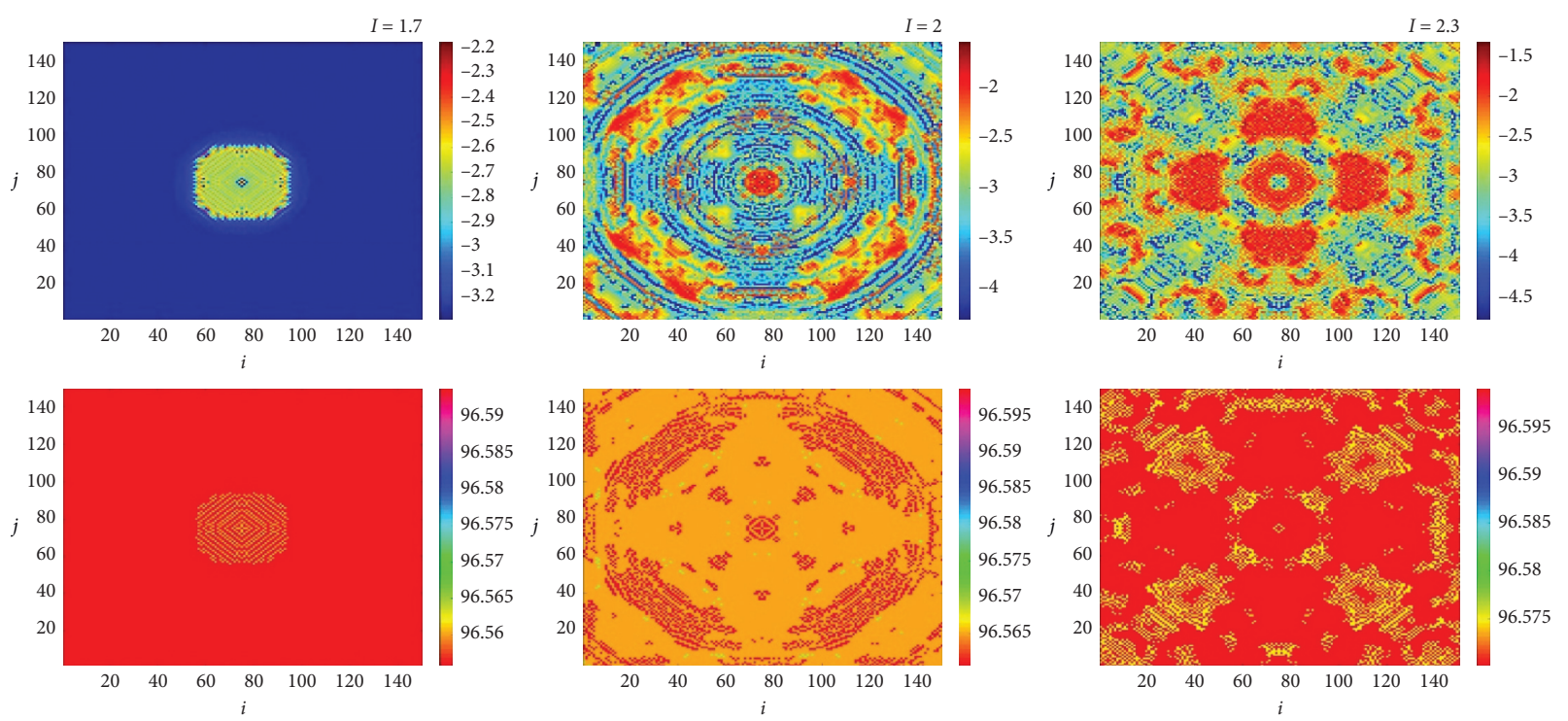

(a)
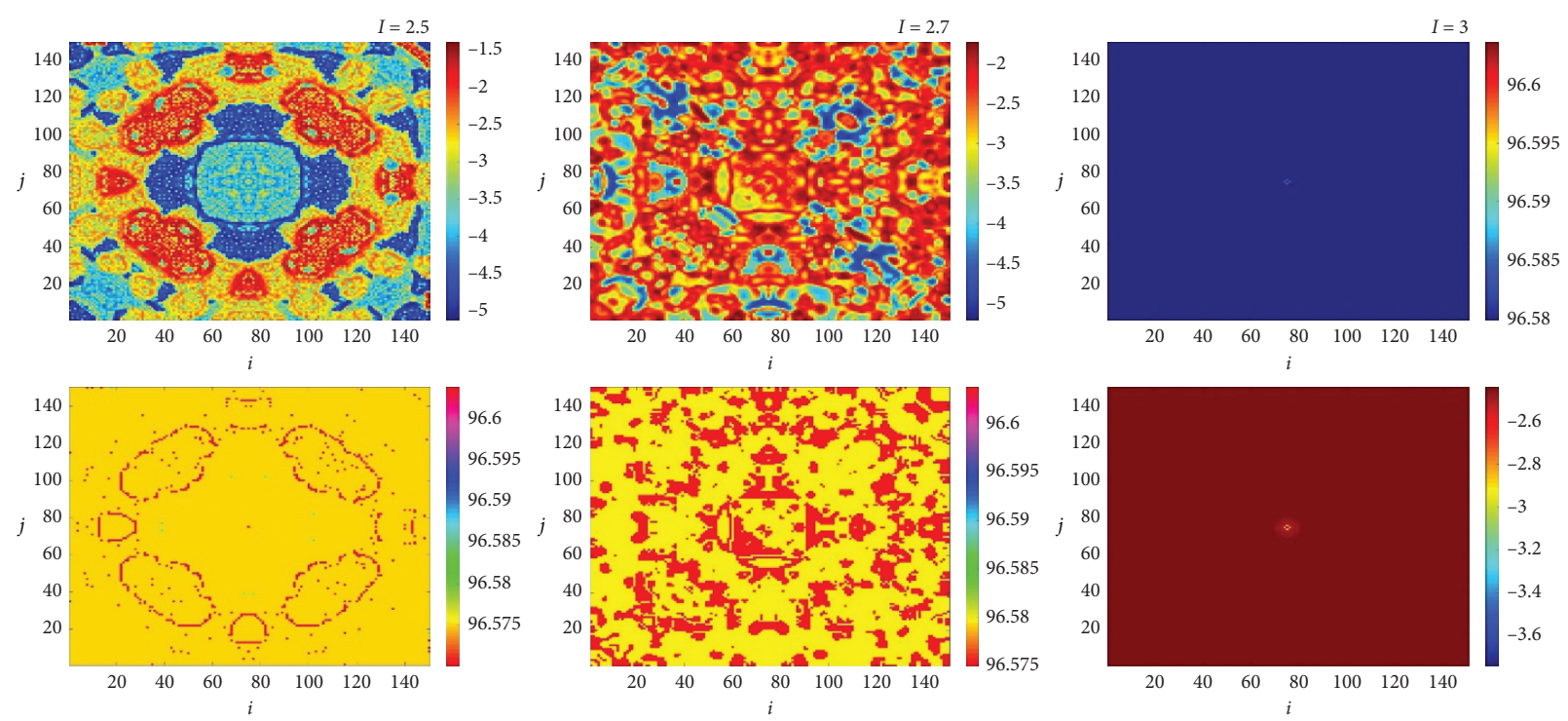

(b)

Figure 6: (a) Dynamics of the network for various values of the bias current amplitude (I). (b) The corresponding periodicity of each node for different $I$. The bias current frequency is kept as $\omega=1.3$.

In Figure 5, we consider the frequency of bias current to be $\omega=0.0001$, and we vary the amplitude of the bias current. For $I<1$, the network goes unbounded, and hence, we have not showed those snapshots. For $I=1$, the network shows broken target waves which are obstructed by the low periodic nodes shown by the yellow-red regions in Figure 5(b). As the bias current is time-varying, the spiral waves seen in Figure 2 for $I=1$ are partially destroyed when a timevarying bias current is applied even though the frequency of bias current is as low as $\omega=0.0001$. This is because that the JJM shows infinite coexisting attractors when a AC bias current is applied [26]. Furthermore, as we have chosen random initial conditions for the network ranging between $[0,5]$, the possibility of coexistence is more considering there exist multiple attractors in this range as shown in [26]. When we increase $I$ further, the nodes are highly incoherent with different periods of oscillations as shown in Figure 5. Hence, uniform pattern formation is now seen for the range $1.2 \leq I \leq 1.9$, but for $I=2$, a symmetric pattern exists which is due to the individual nodes with low period oscillations which confirms that the JJM is exiting the complex chaotic phenomenon. Further increasing $I$ shows that the period bandwidth (refer to the colour bar in Figure 5(b)) keeps reducing and finally reaches a bandwidth of 35 confirming that the nodes are coming into synchrony. This partial coherency is shown with the patterns containing multiple small pools of incoherent nodes and coherent nodes.

For the bias current frequency $\omega=1.3$, the network goes unbounded for values of bias current amplitude $I<1.7$, and this confirms that the periodic bias current plays a vital role 

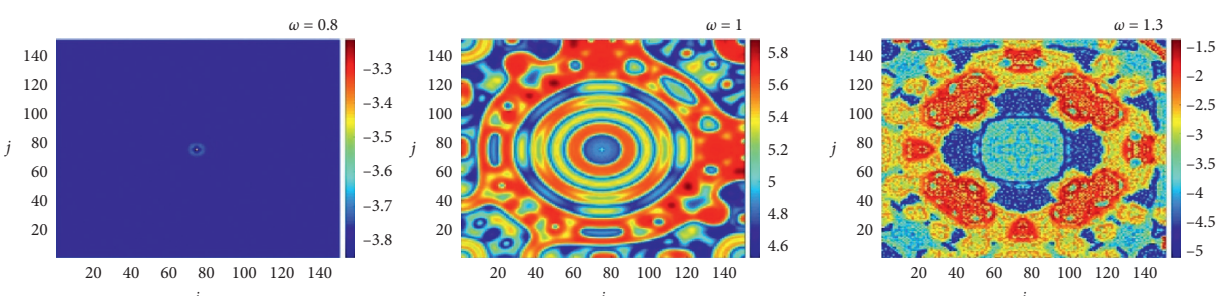

(a)
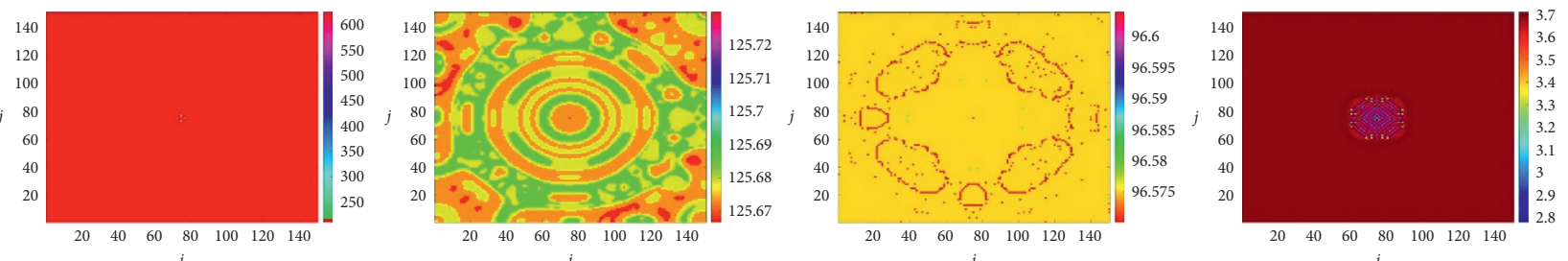

(b)

Figure 7: (a) Dynamics of the network for various values of the bias current frequency ( $\omega$ ). (b) The corresponding periodicity of each node for different $\omega$. The bias current frequency is kept as $I=2.5$.

in determining the dynamical properties of the JJM [26]. For $I=1.7$, there are no oscillations in the network as can be seen from the period plot in Figure 6 which shows a total period bandwidth of 0.03 . The applied stimulus tried to excite the local nodes around the centre, but due to the lack of self-excited oscillations in the nodes due to the current amplitude, the wave propagation diminishes. Hence, we increase the value of $I$ to see the network behaviour. For $I=2$, the nodes are now excited to show periodic bursting which now encourages the wave propagation. This can be seen by the formation of local pools of low period oscillations shown in red in both the spatiotemporal snapshot and the periodic plot. Having low period of oscillation means that the nodes are in chaotic regions and thus show self-excited oscillations. The local nodes forming small groups are due to the coherency behaviour between them which are shown by similar colour in Figure 6. For $I=2.5$, we could see a perfect correlation between the snapshots and the periodicity plots confirming the pattern formations are due to the high-amplitude spiking of the nodes. Further increasing the current amplitude results in diving of these large-amplitude groups which are soon driven unbounded by the bias current amplitude. This confirms that the periodic bias current plays a significant role in the network performance of the JJM.

In the second part of our investigation, we keep the amplitude of the bias current fixed and vary the frequency of the bias current $(\omega)$ and capture the wave propagation of the applied stimulus as in Figure 7. We could show that the pattern formations in the network are largely due to the grouping of similar period nodes or in other works' coherent nodes. These coherent nodes are excited by the applied stimulus to different amplitudes and hence have different patterns (colours) as confirmed from the periodicity plots (Figure 7(b)). Increasing $\omega$ after 1.3 results in the nodes being completely synchronised and thus does not have irregular or complex bursting which results in the nodes being unsupportive or nonreactive to the stimulus. Hence, the excitation of the nodes is only seen near the centre where the stimulus is applied. We could also correlate this with the discussion in [26], where it was shown that, for $\omega>1.3$, the JJM exits bursting oscillations.

\section{Conclusion}

The collective dynamics of a Josephson junction chaotic oscillator considering feedback flux coupling is investigated. A simple lattice array of the JJM-based oscillator is constructed, and we have assumed no-flux boundary conditions. We first applied a DC bias current, and a sinusoidal stimulus is applied to the centre of the network. The network shows nodal spiral waves which are seen for larger amplitudes of the stimuli. Comparing the network snapshots with the periodicity plots, we could see that the spiral waves are exhibited when the period difference between the nodes is less or, in other words, when most of the nodes are in the homogeneous state. When we applied very low-amplitude stimuli, the period difference increases and obstructs the target wave to propagate in higher velocities and thus eliminates the spiral wave transformations in the network. Hence, we could show that low-amplitude stimulus force eliminates the spiral waves, while the highamplitude stimuli induce them. Now, in the second discussion, we have considered AC bias current (sinusoidal). For high frequency of the bias current, the network forms unique patterns which can be compared with the respective periodic plots, and we could say that the boundaries of the formed patterns are defined by low period nodes. These low period nodes split the networks into pools of small energy areas which collectively defined the local performance. In other words, the network forms multiple smaller areas of homogeneous nodes. When we decrease the bias current frequency to low (0.0001), the network shows small turbulent areas which are soon destroyed by the medium 
oscillations. When the nodes are in the chaotic state, the network shows patterned areas confirming pooled oscillations, whereas when the nodes are in the periodic state, the wave propagation is completely terminated. This was not the case in DC bias as when the nodes are in the periodic state, the network shows spiral waves which convert into unique patterns when the nodes enter chaos. As we mentioned, the JJM shows infinite coexisting attractors when excited by AC bias current, and to understand the influence of different attractors (initialised by different initial conditions), we studied the network behaviour for various values of initial conditions. To our surprise, the network behaviour does not change and remains in the same pattern. This confirms that the nodes synchronise and form a homogeneous medium whatever be the attractor type. Hence, the wave propagation behaviour of the network rather depends on the overall synchronisation but not the local coupling alone.

\section{Data Availability}

No data were used to support this study.

\section{Conflicts of Interest}

The authors declare that there are no conflicts of interest regarding the publication of this paper.

\section{References}

[1] G. Zhang, J. Ma, A. Alsaedi, B. Ahmad, and F. Alzahrani, "Dynamical behavior and application in Josephson Junction coupled by memristor," Applied Mathematics and Computation, vol. 321, pp. 290-299, 2018.

[2] A. A. Elsadany, A. M. Yousef, and A. Elsonbaty, "Further analytical bifurcation analysis and applications of coupled logistic maps," Applied Mathematics and Computation, vol. 338, pp. 314-336, 2018.

[3] T. Hongray, J. Balakrishnan, and S. K. Dana, "Bursting behaviour in coupled Josephson junctions," Chaos: An Interdisciplinary Journal of Nonlinear Science, vol. 25, no. 12, Article ID 123104, 2015.

[4] Y. Liu, G. Ren, P. Zhou, T. Hayat, and J. Ma, "Synchronization in networks of initially independent dynamical systems," Physica A: Statistical Mechanics and Its Applications, vol. 520, pp. 370-380, 2019.

[5] F. Wu, P. Zhou, A. Alsaedi, T. Hayat, and J. Ma, "Synchronization dependence on initial setting of chaotic systems without equilibria," Chaos, Solitons \& Fractals, vol. 110, pp. 124-132, 2018.

[6] L. Pivik, "Autowaves and spatial-temporal chaos in CNNs-Part I: a tutorial," IEEE Transactions on Circuits and Systems I, vol. 42, no. 12, pp. 638-649, 1995.

[7] G. Hu, J. Xiao, L. O. Chua, and L. Pivka, "Controlling spiral waves in a model of two-dimensional arrays of chua's circuits," Physical Review Letters, vol. 80, no. 9, p. 1884, 1998.

[8] F. H. Samie, R. Mandapati, R. A. Gray et al., "A mechanism of transition from ventricular fibrillation to tachycardia," Circulation Research, vol. 86, no. 6, pp. 684-691, 2000.

[9] F. Samie and J. Jalife, "Mechanisms underlying ventricular tachycardia and its transition to ventricular fibrillation in the structurally normal heart," Cardiovascular Research, vol. 50, no. 2, pp. 242-250, 2001.

[10] K. Rajagopal, Z. Wei, I. Moroz, A. Karthikeyan, and P. Duraisamy, "Elimination of spiral waves in a one-layer and two-layer network of pancreatic beta cells using a periodic stimuli," Chaos, Solitons \& Fractals, vol. 139, Article ID 110093, 2020.

[11] P. S. Hagan, "Spiral waves in reaction-Diffusion Equations," SIAM Journal on Applied Mathematics, vol. 42, no. 4, pp. 762-786, 1982.

[12] M. C. Cross and P. C. Hohenberg, "Pattern formation outside of equilibrium," Reviews of Modern Physics, vol. 65, no. 3, pp. 851-1112, 1993.

[13] S. Sinha, J. Saramaki, and K. Kaski, "Emergence of self-sustained patterns in small-world excitable media," Physical Review E, vol. 76, Article ID 015101, 2007.

[14] Q. Wang, M. Perc, Z. Duan, and G. Chen, "Delay-enhanced coherence of spiral waves in noisy Hodgkin-Huxley neuronal networks," Physics Letters A, vol. 372, no. 35, pp. 5681-5687, 2008.

[15] K. Rajagopal, F. Parastesh, H. Azarnoush, B. Hatef, S. Jafari, and V. Berec, "Spiral waves in externally excited neuronal network: solvable model with a monotonically differentiable magnetic flux," Chaos: An Interdisciplinary Journal of Nonlinear Science, vol. 29, no. 4, Article ID 043109, 2019.

[16] B. Hu, J. Ma, and J. Tang, "Selection of multiarmed spiral waves in a regular network of neurons," PLoS One, vol. 8, no. 7, Article ID e69251, 2013.

[17] X. Wu and J. Ma, "The formation mechanism of defects, spiral wave in the network of neurons," PLoS One, vol. 8, no. 1, Article ID e55403, 2013.

[18] G. Zhang, F. Wu, T. Hayat, and J. Ma, "Selection of spatial pattern on resonant network of coupled memristor and Josephson junction," Communications in Nonlinear Science and Numerical Simulation, vol. 65, pp. 79-90, 2018.

[19] H. Li, Y. Yang, Li Wen, S. He, and C. Li, "Extremely rich dynamics in a memristor-based chaotic system," The European Physical Journal-Plus, vol. 135, p. 579, 2020.

[20] K. Rohlf, L. Glass, and R. Kapral, "Spiral wave dynamics in excitable media with spherical geometries," Chaos: An Interdisciplinary Journal of Nonlinear Science, vol. 16, no. 3, Article ID 037115, 2006.

[21] D. Gonze, S. Bernard, C. Waltermann, A. Kramer, and H. Herzel, "Spontaneous synchronization of coupled circadian oscillators," Biophysical Journal, vol. 89, no. 1, pp. 120-129, 2005.

[22] I. Aranson, D. Kessler, and I. Mitkov, "Drift of spiral waves in excitable media," Physica D: Nonlinear Phenomena, vol. 85, no. 1-2, pp. 142-155, 1995.

[23] V. S. Zykov, "Kinematics of rigidly rotating spiral waves," Physica D: Nonlinear Phenomena, vol. 238, no. 11-12, pp. 931-940, 2009.

[24] X. Huang, W. C. Troy, Q. Yang, H. T. Ma, C. R. Laing et al., "Spiral waves in disinhibited mammalian neocortex," Journal of Neuroscience, vol. 24, no. 44, pp. 9897-9902, 2004.

[25] X. Huang, W. Xu, J. Liang, K. Takagaki, X. Gao, and J.-Y. Wu, "Spiral wave dynamics in neocortex," Neuron, vol. 68, no. 5, pp. 978-990, 2010.

[26] B. Ramakrishnan, D. Ali, K. Rajagopal, and A. Akgul, "Infinite attractors in a chaotic circuit with exponential memristor and Josephson junction resonator," AEU-International Journal of Electronics and Communications, vol. 123, Article ID 153319, 2020. 
[27] W. G. Choe and S. Kim, "Spatiotemporal dynamics of domain-wall solitons in the fully frustrated Josephson-junction ladder arrays," Physical Review B, vol. 53, p. R502, 1996.

[28] F. Xie and A. Hilda, "Cerdeira, clustering bifurcation and spatiotemporal intermittency in RF-driven Josephson junction series arrays," International Journal of Bifurcation and Chaos, vol. 8, no. 8, pp. 1713-1718, 1998. 